\title{
Arousal and short-term memory: Effects of caffeine and trial spacing on delayed-alternation performance
}

\author{
WILLIAM S. TERRY and SANDRA G. ANTHONY \\ University of North Carolina, Charlotte, North Carolina 28223
}

\begin{abstract}
Two experiments tested the prediction of Kesner's (1973) parallel memory-stores theory that arousal reduces retention in short-term memory. Using rats as subjects, the effect of caffeine on delayed-alternation performance in a spatial discrimination was investigated. Trial spacing was also manipulated, both alone and in combination with the drug variable. The results showed that, with massed trials, caffeine facilitated alternation at the short delay and inhibited it at the longer delays tested. Using spaced trials, caffeine decreased alternation at all delays. Spaced trials produced overall superior performance as compared with massed trials. This pattern of results is consistent with the view that caffeine reduces short-term retention but, paradoxically, can also increase performance under massed trials by decreasing proactive interference (i.e., retention) from earlier trials.
\end{abstract}

The present experiments investigated the effect of caffeine-induced arousal on performance in a task frequently used to index short-term memory (STM) in animals, the delayed-alternation task. Current theories of animal memory often make the distinction between a transient STM and a more permanent longterm memory (LTM) (e.g., John, 1967; McGaugh \& Dawson, 1971; Wagner, Rudy, \& Whitlow, 1973). While there is often agreement as to the general characteristics and tasks sensitive to each type of memory, the various models can differ with respect to the hypothesized relationship of STM to LTM. Specifically, certain theories suggest a sequential processing of information from STM into LTM. Thus, the strength of representation stored in the latter would be a function of the duration of processing (e.g., rehearsal or consolidation) that had occurred in STM (e.g., John, 1967; Wagner et al., 1973). Alternatively, Kesner (1973) has suggested that STM and LTM are parallel stores, in that information can be independently processed in each (although maintenance in STM can add representation back into the LTM formation system). According to this parallel memory-stores model, variables that facilitate the formation of LTM do not necessarily also facilitate retention in STM, and vice versa.

One such variable of present concern is the administration of pharmacological agents that increase the arousal level of the organism. Kesner argues that drugs such as caffeine can enhance the acquisition of traces in LTM but decrease the strength of representation in STM (by increasing the rate of decay

Address reprint requests to W. S. Terry, Department of Psychology, University of North Carolina at Charlotte, UNCC Station, Charlotte, North Carolina 28223. from STM). This theory can be contrasted with one form of a sequential theory of the memory stores which suggests that such drugs are effective in promoting LTM by their facilitation of processing in STM (e.g., Petrinovich, Bradford, \& McGaugh, 1965). It is generally acknowledged that stimulants such as caffeine do indeed facilitate acquisition and longterm retention (e.g., Paré, 1961). There is much less data concerning their effects on short-term retention. In one case, Jarvik (1969) found that large doses of caffeine $(40-80 \mathrm{mg} / \mathrm{kg})$ had only a small decremental effect on performance of rhesus monkeys in a delayed-response task. Petrinovich et al. (1965) found that strychnine enhanced retention over long delays in a conditioned delayed-alternation task. However, the retention intervals that benefited from the drug $(5-8 h)$ greatly exceeded most estimates of the duration of retention in STM, and it is likely that the results actually reflect LTM. In a task closely related to the present one, Hughs and Greig (1975) found that caffeine (and certain other drugs) reduced the tendency of ferrets to alternate spontaneously. It should be noted that, in many cases, facilitation of short-term retention by stimulants is inferred from the enhanced long-term learning that occurs when the drugs are given posttrial (e.g., Dawson \& McGaugh, 1973).

The present experiments sought to assess the effects of caffeine on the postacquisition performance of rats in a delayed-alternation task. Caffeine was selected as one of the central nervous system stimulants whose dominant site of action seems to be the reticular activating system (Krikstone \& Levitt, 1975), which Kesner's (1973) model suggests is implicated in the arousal process. The alternation task is often used as a measure of STM (e.g., Gordon, 
Brennan, \& Schlesinger, 1976; Roberts, 1974). The first experiment compared presession injections of caffeine and saline on alternation following several delay intervals, while in the second experiment, the variable of intertrial interval (ITI) was added. Although the studies were directed toward assessing central nervous system arousal and STM, it is possible that the caffeine could have affected some other stage of memory processing (such as encoding or retrieval) or nonmemorial variables.

\section{EXPERIMENT 1}

\section{Method}

Subjects. The subjects were eight male Sprague-Dawley rats, approximately 100 days old. Two other subjects were dropped due to either illness or failure to learn the initial alternation task. All subjects were individually housed, given free access to water, and were put on a $23.5 \mathrm{~h} /$ day food-deprivation schedule beginning 3 days before the start of training.

Apparatus. A T-maze, with interior dimensions of $10.2 \mathrm{~cm}$ wide $\times 15 \mathrm{~cm}$ high, a start stem length of $59.5 \mathrm{~cm}$, and arm lengths of $61.7 \mathrm{~cm}$, was used. The maze was made of plywood, painted flat black, and had guillotine doors to close off the last $25 \mathrm{~cm}$ of the stem and arms. A 5-cm-diam opaque glass dish was located at the end of each arm to contain the food reinforcements.

Procedure. During the first several days of food deprivation, the subjects were handled daily and each received one 5-min period of adaptation to the maze. Food pellets were available in each arm of the maze at this time.

The subjects were then given six sessions of alternation training. Subjects were taken three or four at a time in their home cages to the testing room, where each received six trials (Days 1 and 6) or eight trials (Days 2-5) per session, with approximately a $90-\mathrm{sec}$ intertrial interval. Each alternation trial consisted of two runs in the maze. On the first run, the subject was forced to enter one arm of the maze (the opposite arm being blocked) and there received two $45-\mathrm{mg}$ Noyes food pellets as reinforcement. The subject was then immediately replaced in the startbox and given a free choice of either arm, with food available only in the arm opposite that entered on the first run. In cases in which errors occurred, free-choice runs were repeated until a correct alternation occurred. The correct arm on a given trial varied randomly between left and right over trials.

Following this initial training, the experimental phase began with the introduction of caffeine and of delay intervals between the forced-and free-choice runs of a trial. On Test Days 1 and 3 , four subjects received intraperitoneal injections of caffeine, and on Days 2 and 4, received saline injections. These designations were reversed for the other four subjects. Within each session, an equal number of $0-, 30-$, and 60 -sec delays were scheduled, the delay being the time between removal from the goal arm on one run and placement in the startbox for the next run of the trial. The two longer delay intervals were spent in the home cages. Nine total trials were run on Days 1 and 2 , and six trials on Days 3 and 4 . Subjects were run in rotation, and the ITI thus averaged about $3 \mathrm{~min}$. The correction procedure was continued during testing.

The drug dosage was $25 \mathrm{mg} / \mathrm{kg}$ of caffeine, using a caffeine, sodium benzoate solution (Chemvet Laboratories) with a concentration of 3.75 grains/cc, which is approximately equal parts of caffeine and sodium benzoate. This dosage is comparable to that previously reported to be effective in enhancing conditioning (Paré, 1961) and postacquisition performance (Jarvik, 1969; Miller \& Miles, 1953) of various trained responses. Control injections were an equivalent volume of physiological saline. The injections were given $15 \mathrm{~min}$ prior to each of the test sessions.
Table 1

Mean Percentages Correct Alternation After Different Retention Intervals as a Function of Prior Injection of Saline or Caffeine

\begin{tabular}{cccc} 
& \multicolumn{3}{c}{ Retention Interval (in Seconds) } \\
\cline { 2 - 4 } Drug & 0 & 30 & 60 \\
\hline Saline & 75.0 & 71.6 & 58.7 \\
Caffeine & 86.6 & 68.7 & 47.5 \\
\hline
\end{tabular}

\section{Results}

Over the last two days of initial training, subjects were correctly alternating on $70.1 \%$ of the trials $(\mathrm{SD}=8.03)$. The results of the test sessions are shown in Table 1, which presents the mean-percentagescorrect alternation on caffeine and saline test sessions at the three delay intervals.

The first point to note is that alternation accuracy decreased as the delay interval was lengthened, consistent with the assumption that STM for the arm first visited on the trial was being assessed.

As to the major variable of interest, caffeine slightly facilitated alternation when there was no delay between runs of a trial (i.e., $0 \mathrm{sec}$ ). However, it decreased performance to a chance level when there was a long delay between the two runs (i.e., $60 \mathrm{sec}$ ). There was no substantial caffeine-saline difference with the intermediate delay interval. Individual subject performance at this delay was extremely variable, with some subjects having scores comparable to their 0 -sec scores, while others were more similar to their 60 sec scores.

An analysis of variance, using the within-subject factors of Drug (caffeine vs. saline) and the two extreme Delay Intervals $(0$ and $60 \mathrm{sec})$, showed the following: a significant Delay effect $[F(1,7)=16.2$, $p<$ $.01]$, a nonsignificant overall Drug effect $(F<1)$, and a significant Drug by Delay interaction $[F(1,7)=$ $5.84, \mathrm{p}<.05]$. Post hoc tests showed that the caffeine and saline conditions did not differ significantly at either delay ( $\mathrm{ts}=1.74$ and 1.68 , respectively). While both drug conditions had significant decreases in alternation from 0 to $60 \mathrm{sec}$, the magnitude of this decrement was greater on the caffeine trials $[t(7)=$ $2.81, \mathrm{p}<.05]$. Thus, the effect of the caffeine was to decrease delay-alternation performance, as compared with the saline condition.

Alternation performance in the caffeine sessions did not vary much across trials within sessions. The mean-percentage-correct responses on the first three vs. the last three trials of the sessions (including one trial at each delay) were $64.5 \%$ and $68.7 \%$, respectively. Values on corresponding trials in saline sessions were $64.6 \%$ and $70.8 \%$.

\section{EXPERIMENT 2}

The results of Experiment 1 demonstrated that caffeine slightly increased an immediate tendency to 
alternate and slightly decreased the tendency to alternate after $60 \mathrm{sec}$, with the overall effect being a greater reduction in alternation than occurred on saline tests. One interpretation of these results is that caffeine enhanced encoding, attention, or some similar mechanism that facilitated immediate performance, but also decreased short-term retention and therefore led to greater forgetting across more substantial delays. These results are consistent with Kesner's (1973) theory in suggesting both the arousing property of caffeine and its decay-enhancing property on STM.

An alternative hypothesis for a 0-sec delay facilitation could be that this also is attributable to forgetting from STM. Correct performance across a daily series of alternation trials could be inhibited by retention of the maze arms entered on previous trials. There is much evidence to support a "proactive interference" mechanism in various delayed-response tasks (e.g., Gordon et al., 1976; Mason \& Wilson, 1974). Variables that induce some forgetting from STM could actually enhance alternation performance by reducing the sources of proactive inhibition.

One variable that could likely influence the amount of intertrial interference is the degree of trial spacing, with massed trials promoting more interference than spaced trials. Jarrard and Moise (1971) have noted a decrement in delayed-response accuracy with massed trials and suggested the retention of earlier trials' stimuli as a source of this interference. Lawicka (1959), using cats in a task requiring retention of one of three spatial positions, noted that the most frequent errors were "perseverative" (i.e., responding to the correct stimulus from the previous trial) and also that the tendency for such errors increased with shorter ITIs. Investigators of the temporal-discrimination hypothesis (e.g., Mason \& Wilson, 1974; Worsham, 1975) have noted the adverse effects of not discriminating in memory which of several prior stimuli occurred most recently as the cue for the current trial.

If caffeine does increase the rate of forgetting, then the above findings suggest that massed trials should show more benefit of the drug in reducing trial-to-trial interference than should occur with spaced trials.

The purposes of the second experiment were to investigate further the caffeine effect observed in Experiment 1 and to assess the effect of trial spacing in combination with the drug variable. Since the literature contains few, if any, reports of ITI effects in delayed-alternation tasks, the first phase of this study was used to determine the parameters leading to a massed-spaced trial difference.

In addition, several changes in procedure were made. A different source of caffeine was used, and in a slightly higher dose, to attempt to increase the caffeine's effects. Also, a modified T-maze was used, both to facilitate running the animals and to ensure the distinctiveness of the two goal arms. Given that drug-effects on conditioning are sometimes susceptible to minor changes in experimental procedure, obtaining a consistent pattern of results across experiments and spacing conditions should enhance the generality of the observed effects.

\section{Method}

Design. All major manipulations were done within subject. The design of Phase 1 was a 2 by 2 by 3 , varying trial spacing (massed and spaced) and delay set $(0,30,60$ or $0,45,120 \mathrm{sec})$ between sessions and the three delay intervals within sessions. Phase 2 employed a similar design, varying trial spacing and drug (caffeine and saline) between sessions and delay interval $(0,45,120 \mathrm{sec})$ within sessions.

Subjects. The subjects were 12 rats, similar to those used in Experiment 1 but about 80 days old. Two subjects were lost due to illness prior to the onset of the experimental phases, leaving 10 subjects from which data are reported.

Apparalus. The subjects were trained in an E-shaped maze, with interior dimensions of $14.1 \mathrm{~cm}$ wide $\times 12.5 \mathrm{~cm}$ high. Each of the three legs of the maze was $31 \mathrm{~cm}$ long and was connected to the $103-\mathrm{cm}$-long stem. The maze was made of plywood, painted flat black, and had guillotine doors at the midpoint of each leg. The center leg was used as the startbox, and the outer legs of the E served as the left and right goalboxes

Procedure. Unless otherwise indicated, the procedures were the same as in Experiment 1. The subjects were food deprived, gentled, and given 4 days of alternation training ( 8 trials/day). Two experimental phases followed.

During Phase 1, subjects received four sessions, one session each on the four conditions produced by the combination of massed and spaced trials with two sets of delay intervals $(0,30$, $60 \mathrm{sec}$ and $0,45,120 \mathrm{sec}$ ). Five subjects received two sessions (one massed and one spaced trial) with the first set of delay intervals, followed by two sessions (one massed and one spaced) with the other set of delays. This sequence was reversed for the remaining subjects. Within each of these two subgroups, the order of massed and spaced trials sessions was counterbalanced so that about an equal number of subjects were run in each of these conditions on a given day.

In the massed trials conditions, each subject received nine daily trials (three at each delay) in immediate succession. In the spaced trials conditions, trials were given in rotation across subjects, with the ITI being determined by the time required to run the other two or three subjects in the squad. With the $0-, 30-, 60-\mathrm{sec}$ delay set, the spaced ITI averaged about $3 \mathrm{~min}$, while this value averaged about $5 \mathrm{~min}$ with the $0-, 45-, 120-\mathrm{sec}$ set. Thus, for the spaced trials conditions, ITI duration was confounded with delay set. The between-subject sequence of delays ensured that each delay occurred once within each block of three subjects, thus holding reasonably constant the values of ITI given above.

During Phase 2, subjects received four test sessions, each defined as one of the cells produced by the combination of drug administration (caffeine and saline) and trial spacing, and now using only $0-, 45-$, and $120-\mathrm{sec}$ delay intervals within each session. Half the subjects received their two massed trials sessions first (three animals injected with caffeine and two with saline on the first session, and the reverse on the on the second), and half received their two spaced trials sessions first (two animals injected with caffeine and three with saline on the first session). The other spacing by drug conditions were then in effect on the last two sessions for the above two subgroups.

During the sessions, subjects received one (no delay) warm-up trial, followed by three trials with each of the $0-, 45-$, and 120 -sec delays between the forced- and free-choice runs of a trial. These delays were presented in a balanced sequence within and across subjects. Massing and spacing of trials were defined by the same procedure used in Phase 1. 
Fifteen minutes prior to each session, subjects were injected intraperitoneally with either a caffeine dosage of $30 \mathrm{mg} / \mathrm{kg}$ of Fisher Scientific caffeine fin a concentration of $15 \mathrm{mg} / \mathrm{cc}$ distilled water) or with an equivalent volume of saline solution.

\section{Results}

The results of Phase 1 , showing the effect of massed vs. spaced trials on delayed-alternation performance, are shown in Figure 1. The percentagecorrect alternation is shown separately for massed and spaced trials for each of the two sets of delay intervals tested. As can be seen, trial spacing had little effect with the shorter delay set (i.e., 0, 30, $60 \mathrm{sec})$. This suggests that the spacing interval used was not sufficient to differentiate it from the massed trials condition. Analysis showed no significant effects of spacing or delay and no interaction of these two variables.

With the longer delay interval set (i.e., 0,45 , $120 \mathrm{sec}$ ), there was a marked spacing effect, with spaced trials producing performance superior to massed trials at each delay interval. Analysis showed a highly significant spacing effect $[F(1,9)=13.98$, $\mathrm{p}<.01$ ] and a marginally significant delay effect $[F(2,18)=3.33, p<.10]$. The interaction of the above two factors was not reliable $(F<1)$.

Due to the confounding of ITI and delay set, it is impossible to say whether the shorter ITI or the particular range of delay intervals $(0$ to $60 \mathrm{sec})$ was dominant in preventing a spacing effect with this combination of parameters. Although performance on certain comparable delays (e.g., 0 and $45 \mathrm{sec}$ ) improved with the longer ITIs of those sessions, performance with a given delay interval can vary across different ITIs (see Spear, 1978, chap. 3).

The results of Phase 2 , showing the combined effects of caffeine and ITI after delays of 0,45 , and $120 \mathrm{sec}$, are shown in Figure 2. Several points should be noted. First, following the saline injections,

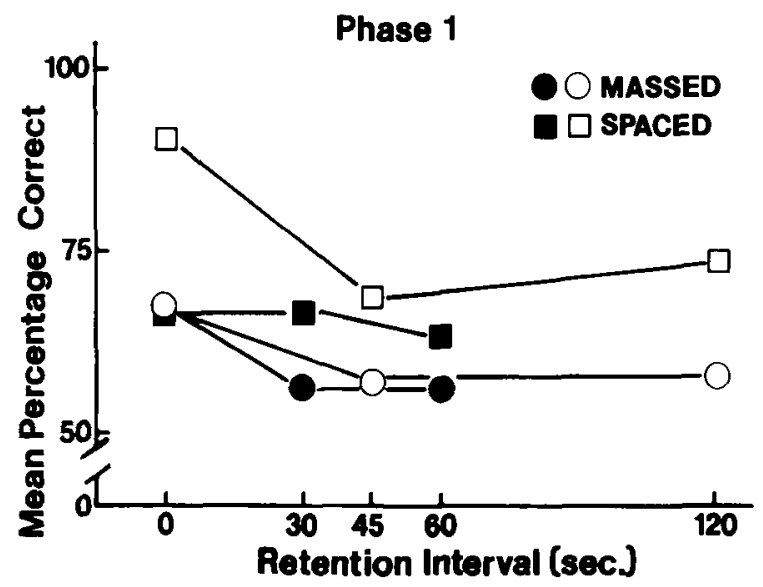

Figure 1. Mean-percentage-correct alternation in sessions testing retention intervals of 0.30 , and 60 sec (filled points), and in sessions testing 0-, 45-, and 120 -sec intervals (open points), as a function of massed or spaced trials within sessions.

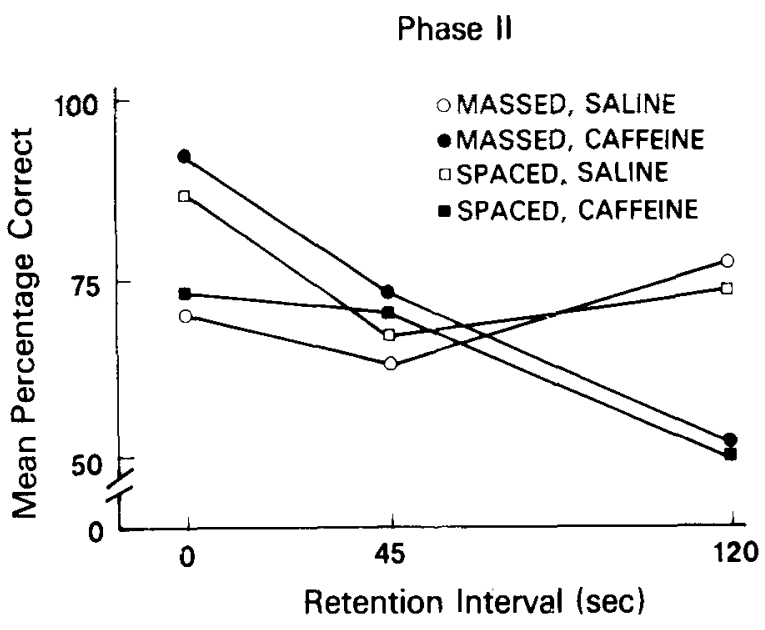

Figure 2. Mean-percentage-correct alternation after 0-, 45-, and 120-sec retention intervals on massed trials sessions following caffeine vs. saline injections and on spaced trials sessions following caffeine vs. saline injections.

spaced trials produced greater alternation accuracy than did massed trials at the 0 - and 45 -sec delays, although not at the longer delay. Thus, the spaced trial facilitation of Phase 1 was partially reproduced. Second, with massed trials, caffeine produced superior performance at the 0 -sec delay and inferior performance at the longest delay, relative to the saline control condition. This is similar to the pattern of results found in Experiment 1. Third, with spaced trials, caffeine did not facilitate immediate-alternation performance; rather, it depressed accuracy below the saline level.

The 45-sec data, in general, showed little discriminability among the several conditions. As in Experiment 1 , the intermediate interval produced substantial variability in performance across subjects, as some animals were comparable here to their 0 -sec performances, while othere were comparable to their 120 -sec levels. Transformation of the data did not reduce the variance sufficiently to show the consistent and otherwise reliable effects at the 0 - and $120-\mathrm{sec}$ delays. Therefore, the first analysis excluded the 45-sec data, while a second analysis is also reported including these values.

A within-subject analysis of variance, using the factors of Trial Spacing, Drug, and Delay Interval $(0$ and $120 \mathrm{sec}$ ) produced the following significant effects: an overall effect of Delay $[F(1,9)=7.41, p<.05]$, a Spacing by Drug interaction $[F(1,9)=20.58$, p < $.01]$, and a Spacing by Drug by Delay interaction $[\mathrm{F}(1,9)=11.02, \mathrm{p}<.01]$. The Drug by Delay interaction was marginally significant $[F(1,9)=4.56, p<.10]$.

Newman-Keuls comparisons of individual means in the three-variable interaction showed, at the 0-sec delay, that the caffeine condition was significantly better when massed trials occurred, but was not different from the saline condition when spaced trials occurred. At the 120 -sec delay, both caffeine conditions were significantly below each saline condition. 
Among the caffeine conditions, massed trials produced superior performance over spaced trials at the 0 -sec delay, but not at $120 \mathrm{sec}$. In comparisons between 0 - and 120 -sec delays, neither saline condition showed a significant change, while both caffeine conditions showed reliable decreases in accuracy (all significant effects, $p<.05$ ).

A comparable analysis of variance, now including data from all three delay intervals, showed a significant effect of Delay $[F(2,18)=4.49, p<.05]$ and a Drug by Delay interaction $[\mathrm{F}(2,18)=6.95, \mathrm{p}<.01]$. Post hoc tests indicated an overall decrement in correct alternation after delays of $\mathbf{4 5}$ and $120 \mathrm{sec}$ (relative to $0 \mathrm{sec}$ ) and that performance was poorer on caffeine trials than on saline trials at 120 -sec delays. Thus, these results at least suggest that delayed alternation may index STM, and also support the prediction from Kesner's (1973) model that caffeine increases forgetting from STM.

An analysis of the data within sessions was done to assess any potential effects of changes in the subjects' motivational levels or variation in the stimulatory effect of the caffeine. In comparing performance on the first three vs. the last three trials of the caffeine sessions (including one trial at each delay), little variation was found. On massed trials days, alternation occurred on $70 \%$ and $76.7 \%$ of the trials of the first and last blocks, respectively. This contrasts with the substantial decrease in accuracy within the saline session, from $73.3 \%$ to $53.3 \%$ on corresponding trial blocks. On spaced trials days, there was little change within either the caffeine session $(59.3 \%$ correct choice on both first and last trial blocks) or the saline session $\mathbf{7 3 3 . 3 \%}$ and $\mathbf{8 3 . 3 \%}$ ). A more detailed analysis within these trial blocks of the Drug by Spacing by Delay combination showed the same pattern of results from Figure 2 on the first and last trials of each type within the sessions. Thus, there were apparently no variations in the level of performance across the time periods necessary for a daily run in the caffeine conditions.

\section{GENERAL DISCUSSION}

In summary, the findings of these two experiments are: (1) a caffeine-related facilitation of alternation performance when minimal retention intervals and massed trials are used, (2) a drug-induced decrement in performance over longer retention intervals or with spaced trials, and (3) a facilitation of delayedalternation performance when trials within sessions are spaced rather than massed.

The decremental effect of caffeine observed here is consistent with Kesner's hypothesis that increased arousal leads to decreased short-term retention. According to this theory, arousal acts to increase the rate of decay of information from STM. Opposite effects are predicted for LTM, as arousal is suggested to facilitate the consolidation process, a component of LTM. Thus, treatments affecting arousal may differentially affect short- and long-term memory. The expected outcome for STM is supported by the present results, while the facilitating effect of arousal on LTM has been demonstrated with caffeine (Paré, 1961) and strychnine (Alpern \& Crabbe, 1972). This differential effectiveness of arousal-inducing agents could be taken to support Kesner's view that STM and LTM are parallel, rather than sequential, systems.

However, other factors may caution acceptance of the arousal-memory relationship. The presently observed effects might be modified if different experimental procedures, drug doses, etc., are used. The literature also shows that drugs having depressant effects may not enhance short-term retention, as Kesner's model suggests should occur. For example, Jarvik (1969) found little effect of several depressants on delayed responding. Alpern and Marriott (1973) found that scopolamine, an anticholinergic agent, decreased accuracy in a successive reversal task. Finally, Alpern and Jackson (1978) have noted that different types of stimulant drugs may have differential effects on the formation and retention of information in memory.

The facilitating effect of caffeine at the shortest delay interval may be due simply to increased attention to the task and better encoding of the stimuli on a trial, or to superior retention in STM over brief delays. However, the fact that the caffeine variable interacted with trial spacing at the shortest delay interval suggests an alternative explanation. Possibly, correct alternation after short ITIs was reduced due to proactive interference from previous trials. If so, performance could be reinstated if the caffeine did induce shortterm forgetting and thus removed the source of proactive interference. With spaced trials, there would be less likelihood of proactive interference, and therefore little or no performance enhancement would be expected to appear in the caffeine condition. Since caffeine was increasing STM loss, even with massed trials performance would drop at the longer intervals as the "correct" stimulus from the first run of the current trial was forgotten.

The superiority of spaced over massed trials in the saline conditions (Experiment 2) is consistent with reports of comparable effects in other short-term memory tasks in animals, for example, delayed matching to sample (Jarrard \& Moise, 1971) and delayed responding (Gleitman, Wilson, Herman, \& Rescorla, 1963). There are several possible mechanisms for this massed trials decrement. The reduced accuracy could be anticipated on the basis of proactive interference of previous stimuli on retention of the more recently presented stimulus. A proactive interference effect has been demonstrated in a task similar to that used here, when rats are given several forced choices to opposite goal arms on the first run of an alternation trial (Gordon et al., 1976). On the other hand, a 
proactive facilitation effect can occur if the rats are given several forced choices into the same arm on the first run of a trial (Roberts, 1974). These findings support the present suggestion that STM on a given trial can be affected by the retention of stimuli from earlier trials.

Alternatively, one could argue that the trial spacing effects are mediated by the capacity to discriminate among many recent memories. According to a temporal discrimination hypothesis (D'Amato, 1973; Worsham, 1975), performance in a delayed-response task with massed trials could be inhibited if the subject cannot discriminate which of several traces in memory is the most recent. When trials are spaced, such traces should be temporally more discriminable in memory. As for the effect of caffeine, one could likely incorporate either a facilitating or inhibiting effect of the stimulant on temporal discrimination into the hypothesis. However, it is not clear how the presently observed interactions are also to be interpreted.

It is possible that test phase performance was affected by generalization decrement. For example, the training phase of Experiment 2 employed spaced trials, with massed trials being introduced only later. This interpretation is made less viable by the fact that one spaced trials condition (the session having 0 -, $30-$, and 60-sec delays) was most comparable in ITI to that used during training, but did not have any effects significantly different from the massed trials condition. In a similar fashion, one could argue that the caffeine decrement also was due to generalization decrement during testing. However, the caffeine facilitation observed in certain conditions would argue against this view.

In contrast with a system of parallel short-and long-term memories are the systems of sequential memories (e.g., Atkinson \& Shiffrin, 1968; Wagner et al., 1973). Within this type of theory, one way of incorporating the facilitative effects of caffeine and other stimulants on LTM is to suggest that the agents also facilitate STM. This interpretation is not supported by the present results. However, the sequential theory is not necessarily invalidated. One could argue that although caffeine reduces the duration of shortterm retention, it increases the effectiveness or quality of whatever short-term processing still occurs. Another possibility is offered by certain sequential theories that postulate different components of STM itself. Thus, one could separate STM from the rehearsal buffer (or rehearsal process) contained within it (Atkinson \& Shiffrin, 1968; Wagner, 1978). Or one could distinguish between a passive "maintenance" rehearsal and a more active "elaborative" rehearsal (Craik \& Lockhart, 1972). In either case, the caffeine could retard one aspect of short-term retention but have a facilitative effect on those aspects (i.e., "rehearsal" or "elaboration") necessary for long- term learning. Such an interpretation could reconcile the present arousal-inhibiting effects on retention with prior observations that "surprising" stimuli (which might be assumed to be more arousing than expected stimuli) are better retained in STM (Terry \& Wagner, 1975).

Another hypothesis for the present results is that the caffeine could be affecting processes other than STM. As with any memory task involving pretrial administration of a drug, it is possible that the present results could be due to encoding or retrieval aspects of memory, or to nonmemory aspects of performance, rather than to the retention mechanisms (Alpern \& Jackson, 1978; Jarvik, 1969). While such interpretation cannot be ruled out here, the present results are at least consistent with several theories of short-term retention.

Finally, it must be acknowledged that the concept of "arousal" itself is apparently not a unitary variable. A given measure of arousal can produce different results with alternative arousal inducers, and different measures can produce conflicting results even in the same task (see Kahneman, 1973). This has led some investigators to distinguish among different kinds of arousal (e.g., Kahneman, 1973; Pribram \& McGuinness, 1975). Any theory concerning the effects of arousal on various memory processes may thus have to provide a more explicit account of the interaction of these variables.

\section{REFERENCES}

Alpern, H. P., \& Crabbe, J. C. Facilitation of the long-term store of memory with strychnine. Science, 1972, 177, 722-724.

Alpern, H. P., \& Jackson, S. J. Stimulants and depressants: Drug effects on memory. In M. A. Lipton, A. D. Mascio, \& K. F. Killam (Eds.), Psychopharmacology: A generation of progress. New York: Raven Press, 1978.

Alpern, H. P., \& Marriot,, J. G. Short-term memory: Facilitation and disruption with cholinergic agents. Physiology \& Behavior, 1973, 11, 571-575.

Atrinson, R. C., \& Shifrin, R. M. Human memory: A proposed system and its control processes. In K. W. Spence (Ed.), The psychology of learning and motivation (Vol. 2). New York: Academic Press, 1968.

Cralk, F. I. M., \& Lockhart, R. S. Levels of processing: A framework for memory research. Journal of Verbal Learning and Verbal Behavior, 1972, 11, 671-684.

D'Aмато, M. R. Delayed matching and short-term memory in the monkey. In G. H. Bower (Ed.), The psychology of learning and motivation (Vol. 7). New York: Academic Press, 1973.

Dawson, R. G., \& McGaugh, J. L. Drug facilitation of learning and memory. In J. A. Deutsch (Ed.), The physiological basis of memory. New York: Academic Press, 1973.

Gleitman, H., Wilson, W. A., Herman, M., \& Rescorla, R. A. Massing and within-delay position as factors in delayedresponse performance. Journal of Comparative and Physiological Psychology, 1963, 56, 445-451.

Gordon, W. C., Brennan, M. J., \& Schlesinger, J. L. The interaction of memories in the rat: Effects on short-term retention performance. Learning and Motivation, 1976, 7, 406-417.

Hughes, R. N., \& GREic, A. M. Spontaneous alternation in ferrets following treatment with scopolamine, chlordiazepoxide, and caffeine. Physiological Psychology, 1975, 3, 155-156. 
J AHHARD. L. E., \& MOISE, S. L. Short-term memory in the monkey. In L. Jarrard (Ed.). Cogninive processes of nonhuman primates. New York: Academic Press, 1971.

Jakik, M. E. Effects of drugs on memory. In P. Black (Ed.), Drugs and the brain. Baltimore: Johns Hopkins University Press. 1969.

Jouv. E. R. Mechanisms of memory. New York: Academic Press, 1967.

Kalineman, D. Altention and effort. Englewood Cliffs, N.J: Prentice-Hall, 1973.

Kesner. R. A neural system analysis of memory storage and retrieval. Psychological Bulletin, 1973, 80, 177-203.

Krikstone, B. J., \& Levitt, R. A. Stimulant and antidepressant drugs. In R. A. Levitt (Ed.), Psychopharmacology: $A$ biological approach. Washington, D.C: Hemisphere, 1975.

LaWicka, W. Physiological mechanisms of delayed reactions Il. Delayed reactions in dogs and cats to directional stimuli. Acta Biologiae Experimentalis, 1959, 19, 199-219.

MASON, M., \& Wilson, M. Temporal differentiation and recognition memory for visual stimuli in rhesus monkeys. Journal of Experimental Psychology, 1974, 103, 383-390.

McGaugh, J. L., \& Dawson, R. G. Modification of memory storage processes. In W. K. Honig \& P. H. R. James (Eds.), Animal memory. New York: Academic Press, 1971.

Mili,er, N. E., \& Miles, W. R. Effect of caffeine on the running speed of hungry, satiated, and frustrated rats. Journal of Comparative Psychology, 1935, 20, 397-412.

PARÉ, W. The effect of caffeine and seconal on a visual discrimination. Journal of Comparative and Physiological Psycholog $v, 1961,54,506-509$.
Petrinovich, L., Bradford, D., \& McGaugh, J. L. Drug facilitation of memory in rats. Psychonomic Science, 1965, 2, 191-192.

Pribram, K. H., \& McGuinness, D. Arousal, activation, and effort in the control of attention. Psychological Review, 1975, 82, 116-149.

Roвe RTS, W. A. Spaced repetition facilitates short-term retention in the rat. Journal of Comparative and Physiological Psychology, 1974, 86, 164-171.

Spe AR, N. E. The processing of memories: Forgetting and retention. Hillsdale, N.J: Erlbaum, 1978.

TERry, W. S., \& Wagner, A. R. Short-term memory for "surprising" versus "expected" unconditioned stimuli in Pavlovian conditioning. Journal of Experimental Psychology: Animal Behavior Processes, 1975, 1, 122-133.

WAgner, A. R. Expectancies and the priming of STM. In S. Hulse, H. Fowler, \& W. Honig (Eds.), Cognitive processes in animal behavior. Hillsdale, N.J: Erlbaum, 1978.

Wagner, A. R., Rudy, J. W., \& Whitlow, J. W. Rehearsal in animal conditioning. Journal of Experimental Psychology, 1973, 97, 407-426. (Monograph)

Worsham, R. W. Temporal discrimination factors in the delayed matching-to-sample task in monkeys. Animal Learning \& Behavior, 1975, 3, 93-97.

(Received for publication July 23, 1979; accepted revision received April 8, 1980.) 